\title{
A new higher order GUP: one dimensional quantum system
}

\author{
Won Sang Chung ${ }^{1, \mathrm{a}}$, Hassan Hassanabadi ${ }^{2, \mathrm{~b}}$ \\ ${ }^{1}$ Department of Physics and Research Institute of Natural Science, College of Natural Science, Gyeongsang National University, \\ Jinju 660-701, Korea \\ ${ }^{2}$ Faculty of Physics, Shahrood University of Technology, Shahrood, Iran
}

Received: 10 December 2018 / Accepted: 23 February 2019 / Published online: 8 March 2019

(C) The Author(s) 2019

\begin{abstract}
In this paper we present a new higher order generalized (gravitational) uncertainty principle (GUP*) which has the maximal momentum as well as the minimal length. We discuss the position representation and momentum representation. We also discuss the position eigenfunction and maximal localization states. As examples we discuss one dimensional box problem and harmonic oscillator problem.
\end{abstract}

\section{Introduction}

Recently, there has been a great interest to study the effects of the Generalized Uncertainty Principle (GUP) [1-31]. Based on a definition of the path integral for string theory, the Polyakov generating functional studied by Konishi, Paffuti, and Provero that they derived the existence of a minimum physical length and a generalized form of the uncertainty principle [10]. On the other hand, Quantum Gravity (QG) theory predicted a minimal length was studying high-energy. In fact, this needs to a generalized in Heisenberg uncertainty principle in order Planck length in quantum mechanics $(\mathrm{QM})$ to leads to this result that there exists a minimal length in QM. Therefore, QM and QG are association (proportional) whit together about exists the minimal length or the generalized uncertainty principle [28]. Many authors have tried to find this difference between QM and QG. Then, Kempf et al. proposed [14] a modified commutation relation between position and momentum that leads to a generalized uncertainty principle (GUP). Then, Kempf introduced a minimal length scale to the mathematical basis of QM $[13,15]$. This leads to different models employing modifications of the canonical commutation relations. Several works have been devoted to performing the uncertainty relations and their measurability bounds in QG. Gedanken (thought) experiments have been proposed to measure the area of apparent horizon of

\footnotetext{
a e-mail: mimip44@ naver.com

be-mail: h.hasanabadi@shahroodut.ac.ir
}

a black hole $[7,8,12]$. Accordingly, a generalization of the uncertainty principle has been concluded. The GUP approach agrees well with the one which is deduced from the String theories $[5,7,8,12,28]$;

$[X, P]=i \hbar\left(1+\beta^{2} P^{2}\right)$.

This gives the uncertainty relation

$\Delta X \Delta P \geq \frac{\hbar}{2}\left[1+\beta^{2}(\Delta P)^{2}\right]$

which suggests the existence of the fundamental minimal length $(\Delta X)_{0}=\hbar \beta$. Here, $\beta^{2}=\beta_{0}^{2} /\left(m_{p} c\right)^{2}$ where $m_{p}$ is the Planck mass with $m_{p} c^{2} \sim 10^{19} \mathrm{GeV}$ and $\beta_{0}$ is of order the unity. Thus we have

$\frac{\beta^{2}}{c^{2}} \sim 10^{-38} \mathrm{GeV}^{-2}$.

It is well known that the GUP (1) gives the minimal length but it does not give the maximal momentum. To incorporate the idea of the maximal momentum, Ali et al. [32-34] have proposed the following modified commutation relation

$$
\begin{aligned}
{\left[X_{i}, P_{j}\right]=} & i \hbar\left[\delta_{i j}-\alpha\left(P \delta_{i j}+\frac{P_{i} P_{j}}{P}\right)\right. \\
& \left.+\alpha^{2}\left(P^{2} \delta_{i j}+3 P_{i} P_{j}\right)\right]
\end{aligned}
$$

where $\alpha=\alpha_{0} / m_{p}=\alpha_{0} L_{p} c / \hbar$ is the GUP parameter, $P^{2}=$ $\sum_{j=1}^{3} P_{j} P_{j}$. The commutation relation (4) is approximately satisfies by the the following representation

$$
\begin{aligned}
X_{i} & =x_{i} \\
P_{i} & =p_{i}\left(1-\alpha p+2 \alpha^{2} p^{2}\right)
\end{aligned}
$$

where $x_{i}$ and $p_{i}$ obeys the the usual commutation relations $\left[x_{i}, p_{j}\right]=i \hbar \delta_{i j}$ and $p=\sqrt{\sum_{j=1}^{3} p_{j} p_{j}}$. The algebra (4) indeed gives the minimal length $\sim \alpha$ and the maximal momentum $\sim 1 / \alpha$ but it is perturbative, i.e., it is only valid for small values of the GUP parameter. 
To overcome these problems, Pedram [20,21] proposed the higher order GUP of the form

$[X, P]=\frac{i \hbar}{1-\beta^{2} P^{2}}$.

This commutation relation agrees with the algebra (1) to the leading order and contains a singularity at $P^{2}=1 / \beta^{2}$. This fact shows that the momentum of the particle cannot exceed $1 / \beta$, which agrees with Doubly Special Relativity (DSR ) [35-38].

The Eq. (7) involves the even terms in $P$ only. In one dimension, the magnitude of the momentum is not $P$ but $|P|=\sqrt{P^{2}}$. Thus, if we are to construct the new higher order GUP, we should use $|P|$ instead of $P$, which makes us find the simpler form than the Eq. (7). In this paper we present a new higher order generalized (gravitational) uncertainty principle (GUP*) in the form $[X, P]=i \hbar /(1-\beta|P|)$ which has the maximal momentum as well as the minimal length.

This paper is organized as follows: In Sect. 2 we present a new higher order generalized (gravitational) uncertainty principle. In Sect. 3 we discuss the position representation and momentum representation. In Sect. 4 we discuss the position eigenfunction and maximal localization states In Sect. 5 we discuss the one dimensional box problem. In Sect. 6 we discussed the harmonic oscillator problem.

\section{A new higher order GUP}

We present a new higher order generalized (gravitational) uncertainty principle (GUP) in the form

$[X, P]=\frac{i \hbar}{1-\beta|P|}, \quad \beta>0$

where $|P|=\sqrt{\left|P^{2}\right|}$, and $\beta=\beta_{0} /\left(m_{p} c\right)$,

$\frac{\beta}{c} \sim 10^{-19} \mathrm{GeV}^{-1}$.

From now on we will call the Eq. (8) GUP*. The Eq. (8) contains a singularity at $|P|=1 / \beta$. This fact shows that the momentum of the particle cannot exceed $1 / \beta$, which agrees with Doubly Special Relativity (DSR ) [35-38].

Now let us check whether the algebra (8) gives the minimal length or not. Expanding the Eq. (8) with respect to $\beta$, we get

$[X, P]=i \hbar\left(1+\beta|P|+(\beta|P|)^{2}+(\beta|P|)^{3}+\cdots\right)$.

The uncertainty relation that arises from GUP* is given by

$$
\begin{aligned}
& (\Delta X)(\Delta P) \geq \frac{\hbar}{2}\left\langle\frac{1}{1-\beta|P|}\right\rangle \\
& =\frac{\hbar}{2}\left\langle 1+\beta|P|+\beta^{2} P^{2}+\beta^{3}|P| P^{2}+\beta^{4}\left(P^{2}\right)^{2}+\cdots\right\rangle
\end{aligned}
$$

$$
\begin{aligned}
\geq & \left.\frac{\hbar}{2}\left[1+\beta\langle|P|\rangle+\beta^{2}(\Delta P)^{2}+\beta^{3}(\Delta P)^{3}+\beta^{4}(\Delta P)^{4}+\cdots\right\rangle\right] \\
\geq & \frac{\hbar}{2}\left[-\beta(\Delta P)+1+\beta(\Delta P)+\beta^{2}(\Delta P)^{2}\right. \\
& \left.\left.+\beta^{3}(\Delta P)^{3}+\beta^{4}(\Delta P)^{4}+\cdots\right\rangle\right] \\
= & \frac{\hbar}{2}\left[-\beta(\Delta P)+\frac{1}{1-\beta(\Delta P)}\right]
\end{aligned}
$$

where we set $\langle P\rangle=0,\langle|P|\rangle \geq 0$ and used the identities

$\left\langle\left(P^{2}\right)^{n}\right\rangle \geq\left(\left\langle P^{2}\right\rangle\right)^{n}$

and

$|\langle(A B+B A)\rangle| \geq 2 \sqrt{\left\langle A^{2}\right\rangle} \sqrt{\left\langle B^{2}\right\rangle}$.

Rewriting the Eq. (15) we get

$(\Delta X) \geq \frac{\hbar}{2(\Delta P)}\left[-\beta(\Delta P)+\frac{1}{1-\beta(\Delta P)}\right]$.

The right hand side of the Eq. (18) has the minimum at $(\Delta P)=1 /(2 \beta)$, hence the minimal length is

$(\Delta X)_{\min }=\frac{3}{2} \beta \hbar$.

On the other hand, and from a physical viewpoint, GUP's are common phenomenological aspects of all promising candidates of quantum gravity. Like Pedram's higher order GUP, the GUP* belongs to a class of higher order GUP which is mathematically well-motivated and non-perturbative. The GUP* gives the different algebraic structure from Pedram's one and has new implications on the Hilbert space representation of quantum mechanics that overcomes some conceptual problems raised in the original GUP (1) such as the divergence of the energy spectrum of the eigenfunctions of the position operator, which will be discussed later.

\section{Representation}

Now let us find two representations for the GUP*.

\subsection{Momentum representation}

The momentum representation for the algebra (8) is

$X=\frac{i \hbar}{1-\beta|p|} \frac{\partial}{\partial p}, \quad P=p$.

The momentum representation acts on the square integrable functions

$\Phi(p) \in \mathcal{L}^{2}(-1 / \beta, 1 / \beta ; d p(1-\beta|p|))$.

The norm of $\Phi$ is given by

$$
\|\Phi\|^{2}=\int_{-1 / \beta}^{1 / \beta} d p(1-\beta|p|)|\Phi(p)|^{2} .
$$


For the operator $A$, the expectation value of $A$ for the wave function $\Phi(p)$ is

$$
\begin{aligned}
\langle A\rangle_{\Phi} & =\langle\Phi(p)|A| \Phi(p)\rangle \\
& =\int_{-1 / \beta}^{1 / \beta} d p(1-\beta|p|) \Phi(p)^{*} A \Phi(p) .
\end{aligned}
$$

The Schrödinger equation reads

$\left[\frac{p^{2}}{2 m}+V\left(\frac{i \hbar}{1-\beta|p|} \frac{\partial}{\partial p}\right)\right] \Phi(p)=E \Phi(p)$.

\subsection{Position representation}

The position representation for the algebra (8) is

$X=x, \quad P=\frac{p}{|p|}\left(\frac{1-\sqrt{1-2 \beta|p|}}{\beta}\right), \quad p=\frac{\hbar}{i} \partial_{x}$.

The position representation acts on the square integrable functions

$\psi(x) \in \mathcal{L}^{2}(-\infty, \infty ; d x)$

and the norm of $\psi$ is given by

$\|\psi\|^{2}=\int_{-\infty}^{\infty} d x|\psi(x)|^{2}$.

For the operator $A$, the expectation value of $A$ for the wave function $\psi(x)$ is

$\langle A\rangle_{\psi}=\langle\psi(x)|A| \psi(x)\rangle=\int_{-\infty}^{\infty} d x \psi^{*}(x) A \psi(x)$.

The Schrödinger equation reads

$\left[\frac{1}{2 m}\left(\frac{1-\sqrt{1-2 \beta|p|}}{\beta}\right)^{2}+V(x)\right] \psi(x)=E \psi(x)$.

\section{Position eigenfunction and maximal localization states}

The eigenvalue problem for the position operator in the GUP* and in the momentum space is given by

$\frac{i \hbar}{1-\beta|p|} \frac{\partial}{\partial p} \psi_{\lambda}(p)=\lambda \psi_{\lambda}(p)$

which gives the normalized position eigenfunction

$\psi_{\lambda}(p)=\sqrt{\beta} \exp \left(-\frac{i \lambda p}{\hbar}\left(1-\frac{\beta}{2}\right)\right)$.

Now we calculate the scalar product of the position eigenfunction

$\left\langle\psi_{\lambda}(p) \mid \psi_{\lambda^{\prime}}(p)\right\rangle=\frac{2 \beta \hbar}{\lambda-\lambda^{\prime}} \sin \left(\frac{\lambda-\lambda^{\prime}}{2 \beta \hbar}\right)$.

Thus, similar to Ref. [14] and Refs. [20,21], the position eigenfunctions are generally no longer orthogonal. In Fig. 1 shows the plot of $y=\left\langle\psi_{\lambda}(p) \mid \psi_{\lambda^{\prime}}(p)\right\rangle$ versus $x=\frac{\lambda-\lambda^{\prime}}{\hbar}$ for

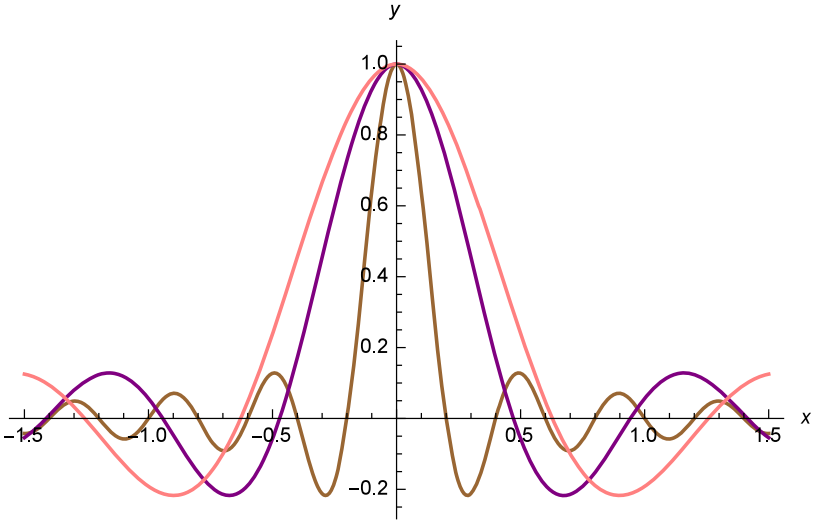

Fig. 1 Plot of $y=\left\langle\psi_{\lambda}(p) \mid \psi_{\lambda^{\prime}}(p)\right\rangle$ versus $x=\frac{\lambda-\lambda^{\prime}}{\hbar}$ for Ref. [14] (brown), Refs. [20,21] (purple) and GUP* (pink)

Ref. [14] (brown), Refs. [20,21] (purple) and GUP* (pink). We know that $\left\langle\psi_{\lambda}(p) \mid \psi_{\lambda^{\prime}}(p)\right\rangle$ for GUP* is similar to Ref. [14] but the zeros are larger than Ref. [14]. This is also less oscillatory than Refs. [20,21].

Now let us consider the maximally localized state $\left|\phi_{\xi}^{m l}\right\rangle$ which is defined through

$\left\langle\phi_{\xi}^{m l}|X| \phi_{\xi}^{m l}\right\rangle=\xi$

and

$\left.(\Delta X)\right|_{\left|\phi_{\xi}^{m l}\right\rangle}=(\Delta X)_{\min }$.

Recall that we chose $\langle p\rangle=0$ for the absolutely smallest uncertainty in position. Let us reconsider the (standard) derivation of the uncertainty relation. The maximally localized state $\left|\phi_{\xi}^{m l}\right\rangle$ obeys

$\left(X-\langle X\rangle+\frac{\langle[X, P]\rangle}{2(\Delta P)^{2}} P\right)\left|\phi_{\xi}^{m l}\right\rangle=0$

where $\langle X\rangle=\xi$. In the momentum space this takes the form of a differential equation

$$
\begin{aligned}
& {\left[\frac{i \hbar}{1-\beta|p|} \partial_{p}-\xi+\frac{i \hbar}{2(\Delta p)^{2}}\right.} \\
& \left.\quad\left(-\beta \Delta p+\frac{1}{1-\beta \Delta p}\right) p\right] \phi_{\xi}^{m l}(p)=0 .
\end{aligned}
$$

If we chose $\Delta p=1 / 2 \beta$, the Eq. (36) is solved into

$$
\begin{aligned}
\phi_{\xi}^{m l}(p)= & N \exp \left[-\frac{i}{\hbar} \xi p\left(1-\frac{\beta}{2}|p|\right)\right. \\
& \left.-\frac{3}{2} \beta^{2} p^{2}\left(1-\frac{2 \beta}{3}|p|\right)\right]
\end{aligned}
$$

where where the normalization factor is given by

$$
\begin{aligned}
N^{-2} & =\int_{-1 / \beta}^{1 / \beta} d p(1-\beta|p|) \exp \left[-3 \beta^{2} p^{2}\left(1-\frac{2 \beta}{3}|p|\right)\right] \\
& \approx \frac{0.7691}{\beta} .
\end{aligned}
$$


One can easily check that the maximally localized state obeys the Eq. (22). However, because of the approximation, we have

$\left.(\Delta X)\right|_{\left|\phi_{\xi}^{m l}\right\rangle} \approx 1.2(\Delta X)_{\min }$

which shows an error about 20 percent. Comparing this with Refs. [20,21] we have two times the error given in Refs. $[20,21]$. This results from the fact that we ignored the positive term $\langle|P|\rangle$ in the Eq. (14). This term is dependent on the form of the wave function.

It is worth to mention that, in GUP*, the expectation value of the kinetic energy operator is finite for both position eigenfunction and the maximal localization states. Indeed we have

$\left\langle\psi_{\lambda}(p)\left|\frac{P^{2}}{2 m}\right| \psi_{\lambda}(p)\right\rangle=\frac{1}{12 m \beta^{2}}$

$\left\langle\phi_{\xi}^{m l}\left|\frac{P^{2}}{2 m}\right| \phi_{\xi}^{m l}\right\rangle=\frac{0.1196}{2 m \beta^{2}}$.

For the case of Refs. [20,21] we have

$\left\langle\psi_{\lambda}(p)\left|\frac{P^{2}}{2 m}\right| \psi_{\lambda}(p)\right\rangle_{\text {Pedram }}=\frac{1}{10 m \beta^{2}}$

$\left\langle\phi_{\xi}^{m l}\left|\frac{P^{2}}{2 m}\right| \phi_{\xi}^{m l}\right\rangle_{\text {Pedram }}=\frac{0.1469}{2 m \beta^{2}}$

and for the case of Ref. [14] we have

$\left\langle\psi_{\lambda}(p)\left|\frac{P^{2}}{2 m}\right| \psi_{\lambda}(p)\right\rangle_{K M M}=\frac{1}{2 m \beta^{2}}$

$\left\langle\phi_{\xi}^{m l}\left|\frac{P^{2}}{2 m}\right| \phi_{\xi}^{m l}\right\rangle_{K M M}=\infty$.

These shows that the expectation values of the kinetic energy operator for both position eigenfunction and the maximal localization states is the smallest for GUP*.

The quasiposition wave function $\psi(\xi)$ is defined as

$\psi(\xi)=\left\langle\phi_{\xi}^{m l} \mid \psi\right\rangle$.

Now the transformation of the wave function in the momentum representation into its counterpart quasiposition wave function is

$$
\begin{aligned}
\psi(\xi)== & N \int_{-1 / \beta}^{1 / \beta} d p(1-\beta|p|)\left[\frac{i}{\hbar} \xi p\left(1-\frac{\beta}{2}|p|\right)\right. \\
& \left.-\frac{3}{2} \beta^{2} p^{2}\left(1-\frac{2 \beta}{3}|p|\right)\right] .
\end{aligned}
$$

This relation shows that similar to the ordinary quantum mechanics, the quasiposition wave function of a momentum eigenstate $\psi_{\bar{p}}(p)=\delta(p-\bar{p})$ with energy $E=\bar{p}^{2} / 2 m$ is still a plane wave but with a modified dispersion relation

$\lambda(E)=\frac{\lambda_{\text {ord }}(E)}{1-\frac{\sqrt{2}}{2} \beta \sqrt{m} \sqrt{E}}$

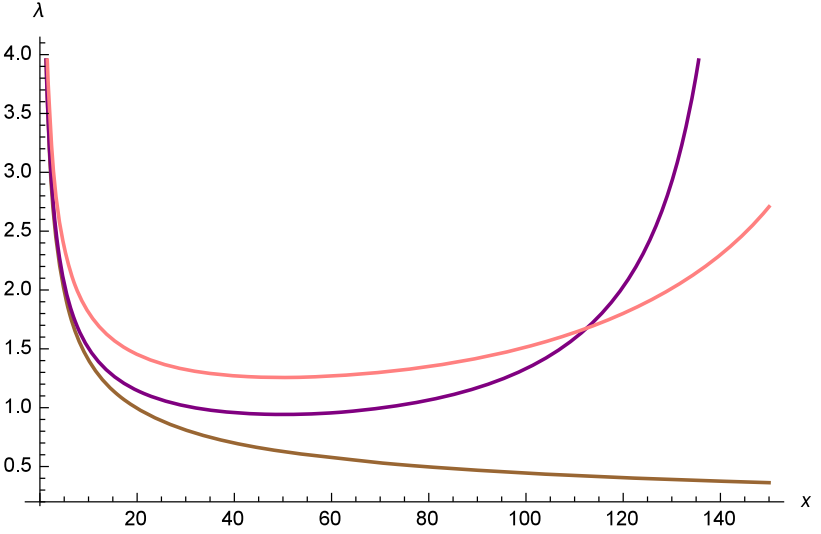

Fig. 2 Plot of $\lambda$ versus $x=m E$ with $\beta=0.1$ for the ordinary case (brown), Refs. [20,21] (purple) and GUP* (pink)

where $\lambda_{\text {ord }}(E)$ is the ordinary wavelength which is obtained from the limit $\beta \rightarrow 0$. Figure 2 shows the plot of $\lambda$ versus $x=m E$ with $\beta=0.1$ for the ordinary case (bown), Refs. [20,21] (purple) and GUP* (pink). Like the Refs. [20,21] the GUP* also has the nonzero minimal wavelength. So the wavelength components smaller than

$\lambda_{0}=\left(\frac{\sqrt{2}}{1-\frac{1}{\sqrt{2}}}\right) \pi \hbar \beta \approx 4.828 \pi \hbar \beta$

are absent in the Fourier decomposition of the quasiposition wave function of the physical states. Therefore, the maximal energy of a momentum eigenstate is

$E_{\text {max }}=\frac{2}{m \beta^{2}}$.

\section{One dimensional Box problem}

Consider a spinless quantum particle with mass $m$ confined to the following one-dimensional box

$V(x)= \begin{cases}0 & (0<x<L) \\ \infty & \text { elsewhere }\end{cases}$

The Schrödinger equation in the position representation reads

$\frac{1}{2 m} P^{2} \psi(x)=E \psi(x)$

The solution of the above equation is

$\psi_{n}(x)=\sqrt{\frac{2}{L}} \sin \frac{n \pi}{L} x$

and the energy is given by

$E_{n}=\frac{\hbar^{2}}{2 m \beta^{2}}\left(1-\sqrt{1-2 \beta\left(\frac{n \pi}{L}\right)}\right)^{2}$. 
For a small $\beta$ we get

$E_{n} \approx \frac{(\hbar n \pi)^{2}}{2 m L^{2}}\left[1+\beta\left(\frac{n \pi}{L}\right)\right]$

which shows that the energy increase due to GUP* effect.

The expectation values of the position and position squared are given by

$$
\begin{aligned}
\langle X\rangle & =\frac{L}{2} \\
\left\langle X^{2}\right\rangle & =L^{2}\left(\frac{1}{3}-\frac{1}{2 n^{2} \pi^{2}}\right)
\end{aligned}
$$

and the expectation values of the momentum and momentum squared are given by

$\langle P\rangle=0$

and we get

$$
\left\langle P^{2}\right\rangle=\frac{\hbar^{2}}{\beta^{2}}\left(1-\sqrt{1-2 \beta\left(\frac{n \pi}{L}\right)}\right)^{2}
$$

where we used

$$
p \sin q x=-i q \cos q x, \quad p \cos q x=-i q \sin q x
$$

$|p| \sin q x=q \sin q x, \quad|p| \cos q x=q \cos q x$.

Thus, the uncertainty relation reads

$$
\Delta X \Delta P=\frac{\hbar L}{\beta}\left(1-\sqrt{1-2 \beta\left(\frac{n \pi}{L}\right)}\right) \sqrt{\left(\frac{1}{12}-\frac{1}{2(n \pi)^{2}}\right)} .
$$

\section{Harmonic oscillator problem}

Now let us discuss the harmonic oscillator for the GUP*. The Hamiltonian for the harmonic oscillator reads

$H=\frac{P^{2}}{2 m}+\frac{1}{2} m w^{2} X^{2}$

\subsection{The semiclassical solution}

The total energy in terms of ordinary variables is

$H=\frac{p^{2}}{2 m}+\frac{m w^{2} x^{2}}{2(1-\beta|p|)^{2}}$.

To find the approximate energy eigenvalues of the above Hamiltonian, we use the Wilson-Sommerfeld quantization rule in the form

$$
\oint x d p=\left(n+\frac{1}{2}\right) h, \quad n=0,1,2, \ldots
$$

which can be written as

$$
\frac{2}{m w} \int_{-z}^{z} d p(1-\beta|p|) \sqrt{z^{2}-p^{2}}=\left(n+\frac{1}{2}\right) h
$$

where

$z^{2}=2 m E$.

Integrating the Eq. (66) we get

$z^{2}-\frac{4 \beta}{3 \pi} z^{3}=2 \hbar m w\left(n+\frac{1}{2}\right)$.

For a small $\beta$ we have

$$
E_{n}^{S C} \approx \hbar w\left(n+\frac{1}{2}\right)+\frac{4 \sqrt{2}}{3 \pi} \beta \sqrt{m}(\hbar w)^{3 / 2}\left(n+\frac{1}{2}\right)^{3 / 2} .
$$

\subsection{Perturbative solution}

In the position representation, the Hamiltonian for the harmonic oscillator reads

$H=\frac{P^{2}}{2 m}+\frac{1}{2} m w^{2} X^{2}$.

Because the Schrödinger equation is not solved in a closed form we will apply the perturbation for a small $\beta$ to GUP* models as follows;

$H=H_{0}+\tilde{H}=H_{0}+\beta H^{\prime}$

where

$H_{0}=\frac{p^{2}}{2 m}-\frac{1}{2} m w^{2} \partial_{p}^{2}$
$H^{\prime}=-\frac{1}{2} m \hbar^{2} w^{2}\left(2|p| \partial_{p}^{2}+\frac{p}{|p|} \partial_{p}\right)$.

Here the momentum wave function and energy for $H_{0}$ are

$H_{0} \psi_{n}(\xi)=E_{n}^{0} \psi_{n}(\xi), \quad n=0,1,2, \ldots$

where

$$
\begin{aligned}
E_{n}^{0} & =\hbar w\left(n+\frac{1}{2}\right) \\
\psi_{n}(\xi) & =\frac{1}{\sqrt{2^{n} n !}}\left(\frac{1}{\pi m \hbar w}\right)^{1 / 4} e^{-\xi^{2} / 2} H_{n}(\xi)
\end{aligned}
$$

and

$\xi=\frac{p}{\sqrt{\hbar m w}}$.

Applying the first order perturbation, we have

$$
\begin{aligned}
\left\langle H^{\prime}\right\rangle_{n}= & -\frac{(m \hbar w)^{3 / 2}}{2^{n+1} m \sqrt{\pi} n !} \int_{-\infty}^{\infty} d \xi e^{-\xi^{2} / 2} H_{n}(\xi) \\
& \times\left(2|\xi| \partial_{\xi}^{2}+\frac{\xi}{|\xi|} \partial_{\xi}\right) e^{-\xi^{2} / 2} H_{n}(\xi) .
\end{aligned}
$$

This integral can be computed numerically. For the first few $n$ 's, we have

$$
E_{0} \approx \frac{\hbar w}{2}+\frac{\beta}{m}\left(\frac{(\hbar m w)^{3 / 2}}{\sqrt{\pi}}\right) \times\left(\frac{1}{2}\right)
$$


Table $12 E_{n} / \hbar w$ for $n=0,1,2,3,4$

\begin{tabular}{llll}
\hline$n$ & $2 E_{n}^{\beta=0} / \hbar w$ & $2 E_{n}^{S C} / \hbar w$ & $2 E_{n} / \hbar w$ \\
\hline 0 & 1 & 1.0042 & 1.0113 \\
1 & 3 & 3.0221 & 3.0226 \\
2 & 5 & 5.0475 & 5.0480 \\
3 & 7 & 7.0786 & 7.0790 \\
4 & 9 & 9.1146 & 9.1150 \\
\hline
\end{tabular}

$E_{1} \approx \frac{3 \hbar w}{2}+\frac{\beta}{2 m}\left(\frac{(m w)^{3 / 2}}{\sqrt{\pi}}\right) \times(2)$

$E_{2} \approx \frac{5 \hbar w}{2}+\frac{\beta}{2 m}\left(\frac{(m w)^{3 / 2}}{\sqrt{\pi}}\right) \times\left(\frac{17}{4}\right)$

$E_{3} \approx \frac{7 \hbar w}{2}+\frac{\beta}{2 m}\left(\frac{(m w)^{3 / 2}}{\sqrt{\pi}}\right) \times(7)$

$E_{4} \approx \frac{9 \hbar w}{2}+\frac{\beta}{2 m}\left(\frac{(m w)^{3 / 2}}{\sqrt{\pi}}\right) \times\left(\frac{163}{16}\right)$.

We know that the energy increases due to GUP*. Figure 1 shows the plot of $E_{n}$ versus $n$ for $\beta=0$ (brown) and $\beta=0.2$ (pink).

6.3 Comparison with the semiclassical solution and perturbative solution

Now let us compare the energy levels for the first few $n$ 's for the ordinary case, semiclassical solution and perturbative solution. Table 1 shows the $2 E_{n} / \hbar w$ with $n=0,1,2,3,4$ for the ordinary case, semiclassical solution and perturbative solution for the dimensionless quantity $\beta \sqrt{\hbar m w}=0.01$. This shows that the energy for the perturbative solution is larger than the semiclassical solution, which results from the fact that we considered only classical region in the semiclassical solution.

\subsection{Classical solution}

Now let us consider the classical solution corresponding to GUP*. The Hamiltonian is

$H=\frac{p^{2}}{2 m}+\frac{1}{2} m w^{2} x^{2}=E$

and the Hamilton equations read

$\dot{x}=\frac{p}{m(1-\beta|p|)}$

$\dot{p}=-\frac{m w^{2} x}{1-\beta|p|}$.

If we set $p_{\max }=2 m E$ we have

$x=\frac{1}{m w} \sqrt{p_{\max }^{2}-p(t)^{2}}$.
Inserting the Eq. (82) into the Eq. (81) we get

$$
w t=-\frac{\beta p}{|p|} \sqrt{p_{\text {max }}^{2}-p(t)^{2}}-\tan ^{-1}\left(\frac{p}{\sqrt{p_{\text {max }}^{2}-p(t)^{2}}}\right) .
$$

The newtonian equation of motion is

$m \ddot{x}=-m W(|\dot{x}|)^{2} x$

where

$W(|\dot{x}|)=w(1+m \beta|\dot{x}|)^{3 / 2}$.

This equation is the Hooke's law with the velocitydependent angular frequency.

\section{Conclusion}

We presented a new higher order generalized (gravitational) uncertainty principle (GUP*) in the form $[X, P]=i /(1-$ $\beta|P|)$ where $|P|=\sqrt{\left|P^{2}\right|}$. For GUP* we explicitly showed that it gives the minimal length. We also obtained the position representation and momentum representation. We discussed the position eigenfunction and Maximal localization states From the comparison with Ref. [14] and Refs. [20,21], we found that the expectation values of the kinetic energy operator for both position eigenfunction and the maximal localization states is the smallest for GUP*. Like the Refs. [20,21] the GUP* was shown to have the nonzero minimal wavelength. We discussed one dimensional box problem where we found that the energy increases due to GUP* effect. We obtained the semiclassical solution and perturbative solution for the harmonic oscillator problem numerically. We found that the energy for the perturbative solution is larger than the semiclassical solution, which resulted from the fact that we considered only classical region in the semiclassical solution.

One can also consider more general case of GUP*,

$$
[X, P]=\frac{i}{(1-\beta|P|)^{N}}, \quad \beta>0, \quad N=1,2, \ldots
$$

where we have

$P=\frac{p}{\beta|p|}[1-(1-\beta(N+1)|p|)]^{\frac{1}{N+1}}$.

But, here, for simplicity, we considered the case of $N=1$ only.

Acknowledgements It is a great pleasure for the authors to thank the referee for helpful comments. This work was supported by the National Research Foundation of Korea Grant funded by the Korean Government (NRF-2015R1D1A1A01057792) and by Development Fund Foundation, Gyeongsang National University, 2018.

Data Availability Statement This manuscript has no associated data or the data will not be deposited. [Authors' comment: All data included 
in this manuscript are available upon request with the corresponding author.]

Open Access This article is distributed under the terms of the Creative Commons Attribution 4.0 International License (http://creativecomm ons.org/licenses/by/4.0/), which permits unrestricted use, distribution, and reproduction in any medium, provided you give appropriate credit to the original author(s) and the source, provide a link to the Creative Commons license, and indicate if changes were made.

Funded by SCOAP ${ }^{3}$.

\section{References}

1. H.S. Snyder, Phys. Rev. 71, 38 (1947)

2. C.N. Yang, Phys. Rev. 72, 874 (1947)

3. C.A. Mead, Phys. Rev. B 135, 849 (1964)

4. F. Karolyhazy, Nuovo Cim. A 42, 390 (1966)

5. G. Veneziano, A stringy nature needs just two constants. Europhys. Lett. 2, 199 (1986)

6. D. Amati, M. Ciafaloni, G. Veneziano, Phys. Lett. B 197, 81 (1987)

7. D.J. Gross, P.F. Mende, Phys. Lett. B 197, 129 (1987)

8. D.J. Gross, P.F. Mende, String theory beyond the Planck scale. Nucl. Phys. B 303, 407 (1988)

9. D. Amati, M. Ciafaloni, G. Veneziano, Phys. Lett. B 216, 41 (1989)

10. K. Konishi, G. Paffuti, P. Provero, Phys. Lett. B 234, 276 (1990)

11. G. Veneziano, Quantum gravity near the Planck scale. In: Proceedings of PASCOS 90, Boston, p. 486 (1990) (unpublished)

12. M. Maggiore, Phys. Lett. B 304, 65 (1993)

13. A. Kempf, Uncertainty relation in quantum mechanics with quantum group symmetry. J. Math. Phys. 35, 4483 (1994). hepth/9311147

14. A. Kempf, G. Mangano, R.B. Mann, Phys. Rev. D 52, 1108 (1995)
15. A. Kempf, On quantum field theory with nonzero minimal uncertainties in positions and momenta. J. Math. Phys. 38, 1347-1372 (1997). hep-th/9602085

16. F. Scardigli, Phys. Lett. B 452, 39 (1999)

17. R.J. Adler, D.I. Santiago, Mod. Phys. Lett. A 14, 1371 (1999)

18. S. Capozziello, G. Lambiase, G. Scarpetta, Int. J. Theor. Phys. 39, 15 (2000)

19. F. Scardigli, R. Casadio, Class. Quantum Gravity 20, 3915 (2003)

20. P. Pedram, Phys. Lett. B 714, 317 (2012)

21. P. Pedram, Phys. Lett. B 718, 638 (2012)

22. M. Bojowald, A. Kempf, Phys. Rev. D 86, 085017 (2012)

23. P. Pedram, Int. J. Mod. Phys. D 19, 2003-2009 (2010)

24. K. Nozari, P. Pedram, EPL 92, 50013 (2010)

25. K. Nozari, M. Moafi, F. Rezaee Balef, Adv. High Energy Phys. 2013, 252178 (2013)

26. K. Nozari, A. Etemadi, Phys. Rev. D 85, 104029 (2012)

27. K. Nozari, P. Pedram, M. Molkara, Int. J. Theor. Phys. 51, 1268$1275(2012)$

28. A. Tawfik, A. Diab, Generalized uncertainty principle: approaches and applications. Int. J. Mod. Phys. D 23.12, 1430025 (2014)

29. H. Shababi, W.S. Chung, Phys. Lett. B 770, 445 (2017)

30. K. Nouicer, Phys. Lett. B 646, 63 (2007)

31. H. Shababi, P. Pedram, W.S. Chung, Int. J. Mod.Phys. A 31, 1650101 (2016)

32. A. Ali, S. Das, E. Vagenas, Phys. Lett. B 678, 497 (2009)

33. S. Das, E. Vagenas, A. Ali, Phys. Lett. B 690, 407 (2010); Erratumibid. 692, 342 (2010)

34. A. Ali, S. Das, E. Vagenas, Phys. Rev. D 84, 044013 (2011)

35. Won Sang Chung, H. Hassanabadi, Phys. Lett. B 785, 127 (2018)

36. J. Magueijo, L. Smolin, Phys. Rev. Lett. 88, 190403 (2002). arXiv:hep-th/0112090

37. J. Magueijo, L. Smolin, Phys. Rev. D 71, 026010 (2005). arXiv:hep-th/0401087

38. J.L. Cortes, J. Gamboa, Phys. Rev. D 71, 065015 (2005). arXiv:hep-th/0405285 